\title{
Fingerprint Image Segmentation Using Haar Wavelet and Self Organizing Map
}

\author{
Sri Suwarno \\ Information Technology Faculty, \\ Duta Wacana Christian University, Yogyakarta \\ Indonesia
}

\author{
Subanar ${ }^{1}$, Agus Harjoko ${ }^{2}$, Sri Hartati ${ }^{3}$ \\ Computer Science Post Graduate Program, Faculty of \\ Mathematics and Natural Sciences, Gadjahmada \\ University, Yogyakarta, Indonesia
}

\begin{abstract}
Fingerprint image segmentation is one of the important preprocessing steps in Automatic Fingerprint Identification Systems (AFIS). Segmentation separates image background from image foreground, removing unnecessary information from the image. This paper proposes a new fingerprint segmentation method using Haar wavelet and Kohonen's Self Organizing Map (SOM). Fingerprint image was decomposed using 2D Haar wavelet in two levels. To generate features vectors, the decomposed image was divided into nonoverlapping blocks of $2 \times 2$ pixels and converted into four elements vectors. These vectors were then fed into SOM network that grouped them into foreground and background clusters. Finally, blocks in the background area were removed based on indexes of blocks in the background cluster. From the research that has been carried out, we conclude that the proposed method is effective to segment background from fingerprint images.
\end{abstract}

Keywords-Fingerprint Segmentation; AFIS; background image; foreground image; Haar wavelet; SOM

\section{INTRODUCTION}

Automatic Fingerprint Identification Systems (AFIS) have been widely used in modern offices, as well as in police departments. The success of AFIS highly depends on the quality of fingerprint images that were inputted to the systems. High quality inputs might be recognized or verified more accurately than low quality inputs. By high quality inputs, we mean fingerprint images that contain a lot of useful information. Unfortunately, fingerprint images are not only composed of useful information but also noises and unnecessary information such as background. In this case, background exists when fingerprint is captured using digital devices that have wider cencor surface than finger area [1] or when latent or rolled fingerprints are scanned using digital devices. To exclude background from computation process, it is necessary to remove background from the image. The process of removing background from image may be defined as segmentation. Zhang et al [2] stated that the goal of segmentation is to discard the background and to reduce the number of false features. This means that segmentation might improve AFIS performance.

Researchers used a number of methods and a variety of features to build fingerprint segmentation algorithms. Segmentation methods might be classified in some ways, and so did feature generation. Based on feature source, segmentation methods can be grouped into block-wise based and pixel-wise based. In block-wise based method, features are extracted from blocks, while in pixel-based method features are extracted from pixels. Commonly, the features extracted from the blocks for this purpose are the values of coherence, mean, variance and field direction. Block-wise segmentation method is fast, but it creates blocking effect in the segmented image edges [3]. To avoid blocking effect, some researchers such as [4] chose pixel-wise method. Theoretically, the method produced finer segmented image, but this method is sensitive to noise and needs longer computation. In [3][5] block-wise and pixel-wise methods are combined to overcome the weaknesses of both methods.

Other researchers proposed classification of segmentation methods from different perspective. Some papers, such as [3][6][7] categorized segmentation methods into supervised and unsupervised, based on the way the decisions are taken. In unsupervised methods, features are extracted from blocks and classification of background and foreground is decided based on a threshold chosen empirically [3]. By choosing an empirical threshold, blocks can be classified as background or foreground. In supervised methods, the decision is made using simple classifier based on training samples [7].

Although some features have been chosen by researchers, finding simple and discriminative features is still a challenging problem. This paper proposed a new segmentation method based on block-wise features generated by Haar wavelet decomposition and using Kohonen's Self-Organizing Maps to classify the blocks into background or foreground. Our method of segmentation would be expected to segment fingerprint images adaptively without user intervention. Although the method produces blocking effect, but the segmented image still reserve a lot of important information that can be used in the next processes, such as classification or recognition.

The rest of the paper is organized as follows. Section 2 discusses feature extraction, Section 3 describes segmentation method, Section 4, presents experiment procedure and some experiment results, Section 5 presents the conclusion of the paper, and finally the future work is presented in Section 6.

\section{FEATURES EXTRACTION}

Theodoridis and Koutroumbas [8] stated that features should be available prior to the design of classifier. Furthermore, they considered that the goal of feature extraction is to generate a vector that uniquely identifies a single object. The size of feature vector should be smaller that the size of the data from which the vector is generated, so that processing of 
feature vectors would be faster than processing the original data itself. Therefore, it is advantageous to select methods that can generate feature vectors as small as possible without losing important information.

There are some choices to generate feature vectors based on the nature of the fingerprint image. Logically, we need to examine what properties of background that differ from foreground. At least there are three properties of background and foreground that can be extracted to form features, namely intensity, homogenity and pattern. Background intensity is usually brighter than foreground intensity. It means that pixel values in the background area are higher than in the foreground area. Related to homogeneity, background area is more homogenous than foreground so that its variant is smaller than foreground variant. Patterns of background and foreground are more difficult to be measured numerically. Some measurements have been proposed to define patterns in fingerprint image, such as orientation or direction of ridges, the number of ridges and the thickness of ridges. These properties have been used extensively, but they are sensitive to noise and need long computation. To overcome the drawbacks we utilized feature generator that indirectly detects intensity, homogeneity and pattern as well. The generator that we chose is Haar wavelet decomposition. We used 2D Haar wavelet decomposition in two levels that decomposed original image into approximation and detail coefficients. Theoretically, all of these coefficients are resulted from linear transformation from the same data. So if we selected only one coefficient, it could reduce computation complexity without degrading the performance. In this method we chose the elements of approximation coefficient as vector feature. This feature consists only four elements. Sometimes the intensity of background pixels are close to furrows' pixels intensity. It means that if the block size is too small, the furrows will be classified as background either. This problem might be solved by considering the size of the furrows. We observed that in 512 dpi fingerprint images, the furrows size are around 6 to 9 pixels. Therefore we chose blocks of size $8 \times 8$ pixels by considering that when this kind of blocks reside in foreground area, they always contain part of furrows, so that those blocks will be classified as foreground.

\section{Segmentation Method}

As mentioned in previous discusion, segmentation may be seen as a classification problem. It is why in [8] and [9] the writers stated that when features have been selected, any classifier can be used to segment background from foreground. Indeed, every classifier has its own advantages and disadvantages. In this research, segmentation process utilized a simple SOM network with only four input nodes and four clusters. Fig. 1 is the diagram of the method that we proposed. It can be seen in Fig 1 that fingerprint image is firsly decomposed using 2D Haar wavelet in two levels. The process produces four coefficients, i.e. approximation, horizontal, vertical and diagonal coefficients. Among these four coefficients, only approximation coefficient is chosen to generate feature vectors.
After this decomposition, the size of the data image is a quarter of the original size. As a result, the size of data blocks in the decomposed image is also a quarter of data blocks size of the original image. Based on this calculation, decomposed image was devided into nonoverlapping blocks of $2 \times 2$ pixels. These blocks are then converted into vectors of 4 elements. All of feature vectors are then fed into SOM network to be clustered.

In this method, the SOM network composed of four input neurons and four clusters. Input of the network are feature vectors resulted from the previous process. In this research, only three alternatives of cluster number, namely two, three and four, were chosen. If the number of cluster is two, theoretically background and foreground blocks would be separated into different clusters. To the contrary, if the cluster number is three or four, there will be more than two alternatives where blocks are clustered. In this research, the number of cluster was tested to find the best performance.

Theoretically, there is no mechanism to determine where a block is grouped. To estimate where background blocks were grouped, we used two considerations,

(1) the number of background blocks is far fewer than the number of foreground blocks, and (2) pixel intensity values of background are higher than pixels intensity of foreground. In SOM training algorithm, higher value inputs tend to be the winners from the first epoch. By considering these two hypothesis, we may predict that background blocks might be cumulated in a cluster that contains fewer number of blocks. Furthermore, background blocks might also be cumulated in cluster with small numbers, namely cluster number 1 or number 2. By using the indexes of blocks in background cluster, the background blocks could be removed from the original fingerprint images.

\section{EXPERIMENT AND RESUlT}

The proposed method was tested using NIST-4 database [10]. In this research, we conducted two experiments by examining the number of epochs and the number of clusters in the SOM training. For the number of epochs, we tested five values, namely $100,125,150,175$, and 200 to find the optimum epoch value.

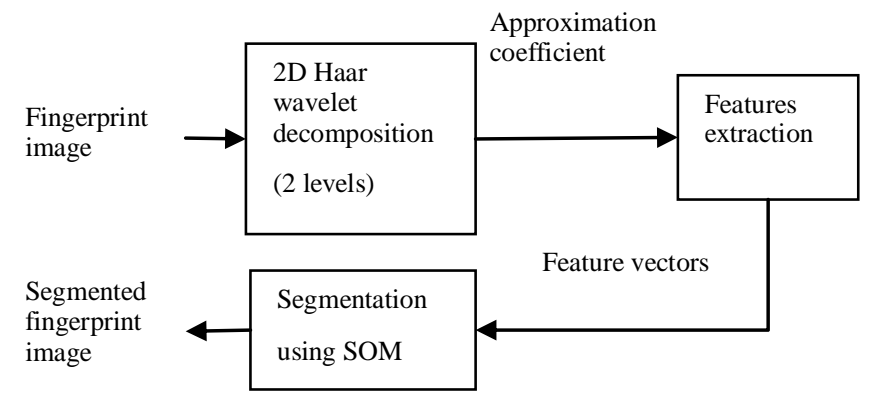

Fig. 1. Diagram of Proposed Method 
TABLE I. Number OF EPOCH Vs Number OF Cluster Member

\begin{tabular}{|c|c|c|c|c|c|c|c|c|c|c|}
\hline & \multicolumn{2}{|c|}{ Epochs $=100$} & \multicolumn{2}{|c|}{ Epochs $=125$} & \multicolumn{2}{|c|}{ Epochs $=150$} & \multicolumn{2}{|c|}{ Epochs $=175$} & \multicolumn{2}{|c|}{ Epochs $=200$} \\
\hline & \multicolumn{10}{|c|}{ Number of cluster members } \\
\hline & Cluster 1 & Cluster 2 & Cluster 1 & Cluster 2 & Cluster 1 & Cluster 2 & Cluster 1 & Cluster 2 & Cluster 1 & Cluster 2 \\
\hline \multicolumn{11}{|l|}{ File Name } \\
\hline F0001_01.tif & 1396 & 2700 & 710 & 3386 & 710 & 3386 & 712 & 3384 & 711 & 3385 \\
\hline F0002_05.tif & 1491 & 2605 & 668 & 3428 & 674 & 3422 & 670 & 3426 & 668 & 3428 \\
\hline F0003_10.tif & 2104 & 1992 & 2111 & 1985 & 2111 & 1985 & 2111 & 1985 & 2111 & 1985 \\
\hline F0004_05.tif & 2037 & 2059 & 2031 & 2065 & 2031 & 2065 & 2031 & 2065 & 2031 & 2065 \\
\hline F0005_03.tif & 1673 & 2423 & 705 & 3391 & 704 & 3392 & 702 & 3394 & 694 & 3402 \\
\hline F0006_09.tif & 1459 & 2637 & 1151 & 2945 & 1151 & 2945 & 1150 & 2946 & 1150 & 2946 \\
\hline F0007_09.tif & 1391 & 2705 & 1216 & 2880 & 1217 & 2879 & 1216 & 2880 & 1216 & 2880 \\
\hline F0008_10.tif & 2319 & 1777 & 2374 & 1722 & 2372 & 1724 & 2373 & 1723 & 2374 & 1722 \\
\hline F0009_08.tif & 1293 & 2803 & 805 & 3291 & 806 & 3290 & 801 & 3295 & 800 & 3296 \\
\hline F0010_10.tif & 1212 & 2884 & 591 & 3505 & 586 & 3510 & 592 & 3504 & 587 & 3509 \\
\hline
\end{tabular}

Furthermore, for cluster numbers we tested three values, namely two, three and four, using the epoch value resulted in the previous experiment.

\section{A. Finding the optimum epoch}

It is desirable that the number of epoch should be as small as possible; however, the SOM training should reach stable condition. To find the optimum number of epoch we tested 100 fingerprint images from NIST-4 database. We used two, three and four clusters as dependent variables to find the optimum epoch numbers. By considering the clarity of visualization, we only presented 10 of them, as seen in Table 1 and Fig. 2. In this experiment, we did not need to examine cluster 2 because it should be foreground cluster. To save the space, we did not present the table results and related figures for experiment with three dan four clusters. The results were almost similar as Table 1 and Fig. 2.

It can be seen in Table 1 that the number of blocks that were cumulated in cluster-1s tend to stable starting from epochs 125 upwards, and Fig. 2 clarify this finding.

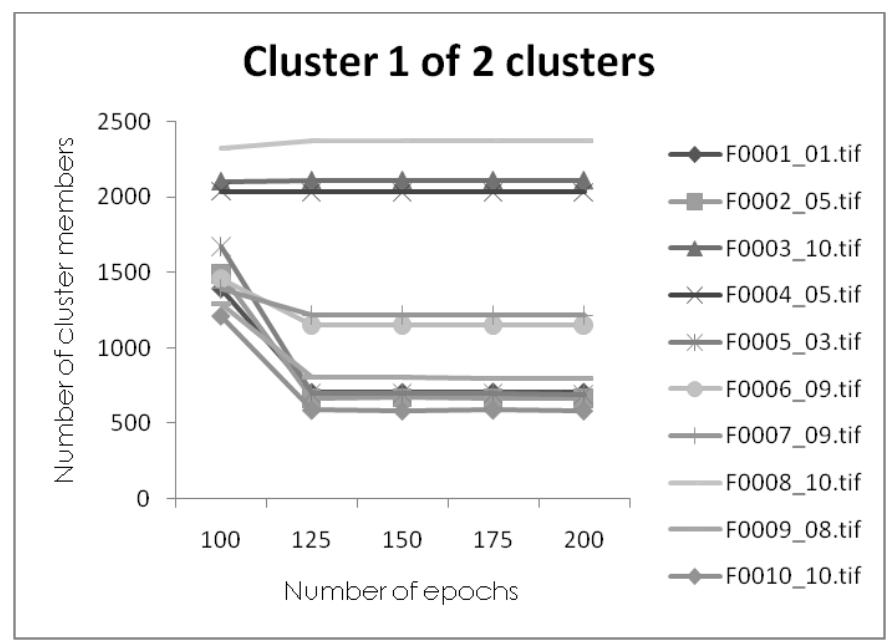

Fig. 2. Number of cluster vs number of epoch for two clusters
From these experiments, it could be concluded that for this method, 125 is the smallest epoch number in order to get converged result.

\section{B. Finding the best cluster number}

After the optimum epoch number was found, namely 125 , then we used this number to find the best cluster numbers. As mentioned in previous explanation, we used two, three and four clusters. It is difficult to measure the best result numerically, so we examined them visually. Fig. 3 and Fig. 4 are the examples of the results.

From these two figures it might be concluded that when the cluster number is two, some blocks inside in the foreground area are considered as background, and showed as white blocks. From our experiments of 100 fingerprint images, we concluded that the best result was when the number of cluster was four.

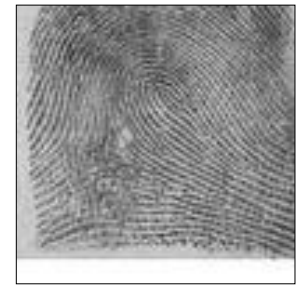

(a)

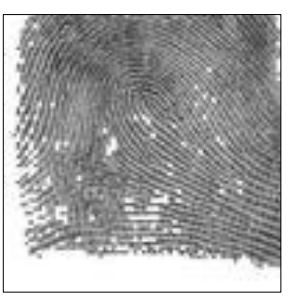

(c)

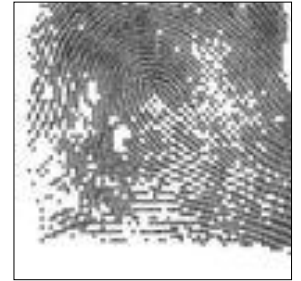

(b)

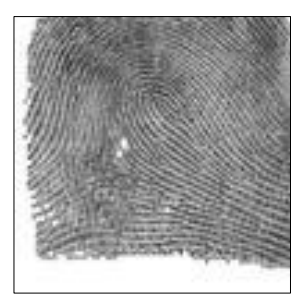

(d)
Fig. 3. Result examples (a) original image (b) cluster number $=2$ (c) cluster number $=3(\mathrm{~d})$ cluster number $=4$ 


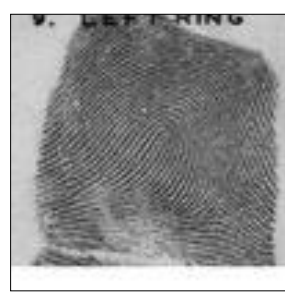

(a)

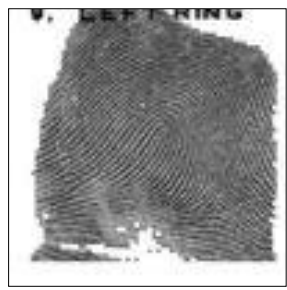

(c)

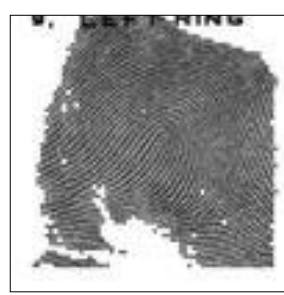

(b)

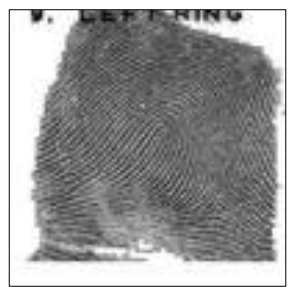

(d)
Fig. 4. Fingerprint with annotation. (a) original image, (b) cluster number $=$ 2 , (c) cluster number $=3$, (d) cluster number $=4$

However, due to the white 32-bit-strip in the bottom of fingerprint images of NIST-4 database, our method using four clusters could not separate background from foreground. In this case, background cluster moved from cluster number 1 to cluster number 2 . Therefore, the algorithm should be modified slightly.

\section{CONCLUSIONS}

We have proposed a new method for fingerprint image segmentation based on block-wise features. We used 100 fingerprint images of NIST-4 database to test the method. The method used Haar wavelet decomposition to generate feature vectors. The vectors were then clustered by Self Organizing Map (SOM) to classify background blocks and foreground blocks. Using block indexes in the background cluster, the background blocks in the original images were removed. The result of our experiments showed that the proposed method is simpler and more adaptive compared to other methods. The method does not need any preprocessing such as enhancement, and it does not need user intervention such as to select threshold either. Based on our experiments, it can be cloncluded that epoch number of 125 upwards would give stable condition of SOM training. Moreover, the optimum number of cluster would be 4 .

\section{FUTURE WORK}

Our future work will continue to implement the method in fingerprint processing applications such as orientation field estimation and classification.

\section{REFERENCES}

[1] H. Jia and P. Tang, "An Improved Fingerprint Image Segmentation Algorithm," in International Conference on Computer Science and Electronics Engineering, 2012, pp. 118-120.

[2] J. Zhang, R. Lai, and C.C.J. Kuo, "Latent Fingerprint Segmentation with Adaptive Total Variation Model," in IAPR International Conference on Biometrics, 2012, pp. 189-195.

[3] S. Zhang, X. Jing, B. Zhang, and S. Sun, "An Adaptive Fingerprint Image Segmentation Algorithm Based on Multipe Features," in Second International Conference on Advanced Computer Control, 2010, pp. 191194.

[4] E. Ruiz-Echartea, M. Morales-Sandoval, M.,A. Nuno-Maganda, and Y.Hernandez-Mier, "A Novel Strategy for Image Segmentation of Latent Fingerprint," in 22nd International Conference on Electrical Communications and Computers, 2012, pp. 196-201.

[5] C. Chen, D. Zhang, L. Zhang, and Y. Zhao, "Segmentation of Fingerprint Image by Using Polarimetric Feature," in International Conference on Autonomous and Intteligent Systems, 2010, pp. 1-4.

[6] M.U Akram, A. Ayaz, and J. Imtiaz, "Morphological and Gradient Based Fingerprint Image Segmentation," in International Conference on Information and Communication Technologies, 2011, pp. 1-4.

[7] J. Ma, X. Jing, Y. Zhang, S. Sun, and H. Huang, "Simple Effective Fingerprint Segmentation Algorithm for Low Quality Images," in IEEE International Conference on Broadband Network and Multimedia Technology, 2010, pp. 855-859.

[8] S. Theodoridis and K. Koutroumbas, Pattern Recognition, 4th ed. London, UK: Academic Press, 2009.

[9] D. Maltoni, D. Maio, A. K Jain, and S. Prabhakar, Handbook of Fingerprint Recognition. London: Springer-Verlag, 2009.

[10] C.I Watson and C.L. Watson, "NIST Special Database 4 Fingerprint Database," National Institute of Standards and Technology Advanced Systems Division, Image Recognition Group, 1992. 